Präv Gesundheitsf 2022 $17: 32-43$ https://doi.org/10.1007/s11553-021-00842-z Eingegangen: 30 . November 2020

Angenommen: 2. März 2021

@ Der/die Autor(en) 2021
Online publiziert: 30. März 2021

Felix Alexander Neumann' (1D - Natascha Mojtahedzadeh ${ }^{2}$. Volker Harth ${ }^{2}$. Stefanie Mache ${ }^{2} \cdot$ Matthias Augustin $^{3} \cdot$ Birgit-Christiane Zyriax $^{1}$

' Universitätsprofessur für Hebammenwissenschaft - Versorgungsforschung und Prävention, Institut für Versorgungsforschung in der Dermatologie und bei Pflegeberufen (IVDP), Universitätsklinikum Hamburg-Eppendorf (UKE), Hamburg, Deutschland

${ }^{2}$ Zentralinstitut für Arbeitsmedizin und Maritime Medizin (ZfAM), Universitätsklinikum HamburgEppendorf (UKE), Hamburg, Deutschland

${ }^{3}$ Institut für Versorgungsforschung in der Dermatologie und bei Pflegeberufen (IVDP), Universitätsklinikum Hamburg-Eppendorf (UKE), Hamburg, Deutschland

\section{Gesundheitsverhalten und -förderung von ambulanten Pflegekräften}

\author{
Eine explorative, qualitative Studie zur \\ aktuellen Situation, potenziellen \\ Hindernissen, Herausforderungen und \\ Lösungsstrategien aus der Sicht von \\ Expert*innen
}

\section{Hintergrund}

Bis 2060 wird laut der Prognose für die Bevölkerungsentwicklung der Anteil der Deutschen im Alter von über 65 Jahren um 5,6 Mio. Menschen anwachsen [23]. Die Zahl an Pflegebedürftigen und benötigtem Pflegepersonal steigt. Schätzungen zufolge fehlen im Jahr 2050 bis $\mathrm{zu} 0,85$ Mio. Fachkräfte in der Pflege [22], die erhöhte gesundheitliche Belastung schlägt sich jedoch schon heute in den Statistiken zur Arbeitsunfähigkeit nieder [25]. Mit durchschnittlich 22,9 krankheitsbedingten Fehltagen in 2018 fallen Pflegekräfte 8 Arbeitstage pro Jahr mehr aus als der bundesweite Durchschnitt aller Berufstätigen. Die Gewinnung von Personal aus dem Ausland, Schaffung finanzieller Anreize und die Gesunderhaltung der Beschäftigten stellen Maßnahmen dar, welche dieser Entwicklung entgegenwirken können [22].

Das Arbeiten im Schichtsystem, Termin- und Leistungsdruck [14] und ein erhöhtes soziales Konfliktpotenzial im
Umgang mit Patienten [27] erschweren gesundheitsförderliche Verhaltensweisen [12] und können infolgedessen $\mathrm{zu}$ gesundheitlichen Beeinträchtigungen führen [21]. Um das hohe Arbeitspensum bewältigen $\mathrm{zu}$ können, wird auf regelmäßige Pausen und somit wichtige Regenerationsphasen verzichtet [28]. Stresserleben als negative Beanspruchungsfolge erhöht das Risiko für psychosomatische und muskuloskelettale Beschwerden [16]. Der Einfluss der Arbeitsbedingungen auf das Ernährungsund Trinkverhalten, die körperliche Aktivität und das Rauchverhalten sowie Auswirkungen auf die Gesundheit ambulanter Pflegekräfte wurden bislang nicht untersucht [19, 20]. Allgemein existiert für die ambulante Pflege insbesondere in kleinen und mittleren Betrieben nur wenig Forschungsliteratur $\mathrm{zu}$ Gesundheitskompetenzen, -verhalten und -förderung [19, 20]. Demzufolge finden sich für ambulant tätige Pflegekräfte in Deutschland keine zielgruppenspezifischen Maßnahmen der betrieblichen Gesundheitsförderung (BGF), was auch auf die Erschwernisse bei der Umsetzung im mobilen Setting zurückzuführen ist [13]. Existierende verhaltenspräventive Gesundheitsförderungsprogramme, entwickelt für die stationäre Pflege, fokussieren zumeist auf Stressbewältigung [19]. Bei der Untersuchung verhältnispräventiver Maßnahmen führte sowohl die Bereitstellung eines Massagestuhls [10], als auch die verbesserte Gestaltung des Pausenraums zu einer signifikanten Reduzierung des Stresserlebens [15]. Allgemein gibt es eine Vielzahl erfolgsgeprüfter Ansätze zur Stressprävention am Arbeitsplatz [2], welche in der Pflege bislang keine Verwendung gefunden haben. Vereinzelt wurden Maßnahmen in der Raucherentwöhnung, Rückenschulung und Seminare zur gesunden Ernährung erfolgreich durchgeführt [19]. Eine erste psychoedukative Interventionsstudie zur Achtsamkeits- und Resilienzförderung in der ambulanten Pflege berichtet von Senkungen der Arbeitsstresswahrnehmung und des Burnout-Risikos [4]. Diese stellt somit einen Meilenstein dar, 


\begin{tabular}{|c|c|c|}
\hline Nr. & Demografie $^{a}$ & Tätigkeitsbereich und pflegebezogene Berufserfahrungen \\
\hline 1 & $w_{1} \geq 41$ & \multirow{3}{*}{$\begin{array}{l}\text { Ambulante Pflege; Leitung Sozialstation (etwa } 50 \text { Beschäftigte) eines } \\
\text { freien Trägers ( }<500 \text { Beschäftigte) mit Schwerpunkt in der ambulan- } \\
\text { ten Kranken- und Altenpflege, sowie Leitung und Stellvertretung eines } \\
\text { ambulanten Intensivpflegedienstes (etwa } 50 \text { Beschäftigte) }\end{array}$} \\
\hline 2 & $m, \geq 41$ & \\
\hline 3 & $\mathrm{~m}, 18-40$ & \\
\hline 4 & $\mathrm{w}, \geq 41$ & \multirow{2}{*}{$\begin{array}{l}\text { Stationäre Pflege; Expert*innen für die zielgruppenspezifische Patienten- } \\
\text { betreuung sowie Weiterbildung und Akademisierung von Pflegekräften } \\
\text { im stationären Setting }\end{array}$} \\
\hline 5 & w, 18-40 & \\
\hline 6 & $\mathrm{w}, \geq 41$ & \multirow{2}{*}{$\begin{array}{l}\text { Funktionäre aus Verbänden der Pflegeberufe als Bindeglied zwischen } \\
\text { Pflegepraxis und Politik }\end{array}$} \\
\hline 7 & $m, \geq 41$ & \\
\hline 8 & $w, 18-40$ & \multirow{3}{*}{$\begin{array}{l}\text { Wissenschaftler*innen mit Projekterfahrung zur Arbeits- und Gesund- } \\
\text { heitssituation von Pflegekräften mit Migrationshintergrund und Gesund- } \\
\text { heitszustand und -verhalten von Auszubildenden in der Pflege }\end{array}$} \\
\hline 9 & $w, 18-40$ & \\
\hline 10 & $m, 18-40$ & \\
\hline
\end{tabular}

deckt jedoch nur wenige Determinanten der Gesundheit ab [5].

\section{Ziel der Arbeit}

Ziel der vorliegenden Studie ist es, auf Basis der Meinung von Expert*innen, Gesundheitsverhalten und -förderung ambulanter Pflegekräfte zu eruieren. Zudem sollen Hindernisse und Herausforderungen für die erfolgreiche Implementierung von BGF sowie potenzielle Lösungsstrategien identifiziert und dabei Erkenntnisse zur Gestaltung zielgruppenspezifischer Maßnahmen gewonnen werden. Folgende Fragestellungen sollen beantwortet werden:

- Wie charakterisieren die Expert ${ }^{*}$ innen das Gesundheitsverhalten ambulanter Pflegekräfte in den Themenbereichen „Ernährungsund Trinkverhalten“, „Rauchen“, „körperliche Aktivität" und „Stress“?

- Welche existierenden BGF-Maßnahmen sind den Expert*innen bekannt?

- Welche Hindernisse und Herausforderungen sind bei der BGFImplementierung zu erwarten?

- Welche Lösungsstrategien im Umgang mit den Hindernissen und Herausforderungen erachten die Expert*innen als sinnvoll?

\section{Material und Methoden}

\section{Studiendesign}

Im Januar 2020 wurde unter Einbezug von 10 Expert $^{*}$ innen aus der Hambur- ger Pflegebranche und -wissenschaft eine Fokusgruppe durchgeführt. Eine Stärke qualitativer Forschungsmethoden und explizit von Fokusgruppen ist es, dass Erwerden können und sich diese Methoden somit besonders für die explorative Phase einer Untersuchung eignen [20]. Die Ergebnisse der Fokusgruppe dienten im nächsten Schritt als Entwicklungsgrundlage für eine quantitative OnlineBefragung.

\section{Rekrutierung und Teilnehmer}

Zunächst wurden im November 2019 Hamburger Expert*innen für Gesundheitsförderung im ambulanten Pflegesektor identifiziert und potenzielle Teilnehmer basierend auf einer internetgestützten Recherche sowie Empfehlungen seitens der Berufsgenossenschaft für Gesundheitsdienst und Wohlfahrtspflege (BGW) zusammengestellt. Die Kontaktaufnahme zu den Expert*innen erfolgte via telefonischer Akquise, per E-Mail als auch persönlich. Die finale Besetzung der Fokusgruppe mit Vertretern aus Pflegepraxis, -verbänden und -wissenschaft verfügte über Expertisen aus verschiedenen Berufsfeldern der Pflege (•Tab. 1) und stärkt die externe Validität der Ergebnisse [18].

\section{Vorgehen}

Die Fokusgruppe wurde mittels eines semistrukturierten Interviewleitfadens durchgeführt. Die Fragestellungen des kenntnisse und Fragestellungen generiert
Interviewleitfadens zum Gesundheitsverhalten ambulanter Pflegekräfte und Maßnahmen der Gesundheitsförderung in der ambulanten Pflege sind im Anhang (•Tab. 2) zur Verfügung gestellt. Um möglichst umfassende Diskussionen anzuregen, wurden offene Fragestellungen verwendet, welche für die Gesundheitsbereiche „körperliche Aktivität", „Ernährung“, „Rauchen“ und „Stress“ wiederholt thematisiert wurden.

Der Leitfaden wurde vorab von einer ambulanten Pflegekraft bestätigt. Ein interdisziplinäres, aus vier Wissenschaftlern bestehendes Studienteam mit langjähriger Erfahrung in der Durchführung qualitativer Forschungsmethoden leitete das Fokusgruppeninterview. Zu Beginn der Fokusgruppe wurden die Teilnehmer über die Studienziele, Diskussionsthemen, Datenschutz und Freiwilligkeit zur Teilnahme informiert. Eine Einwilligungserklärung zu den Teilnahmebedingungen wurde zu Beginn unterzeichnet. Die zweistündige Veranstaltung wurde in Ton aufgezeichnet.

\section{Informations- und Datenverarbeitung}

Zunächst wurde die Tonbandaufzeichnung anhand der vereinfachten Transkriptionsrichtlinien nach Dresing und Pehl [8] verschriftlicht und das Transkript mittels Inhaltsanalyse nach Mayring [17] qualitativ ausgewertet. Der Fokus dieser Auswertungsmethode liegt auf dem Inhalt des Gesagten und unterstützt somit das explorative Forschungsvorhaben [3]. Der validierte, systematische Ansatz der Inhaltsanalyse stärkt zudem die Reliabilität der qualitativen Ergebnisse [17]. Ein Kategoriensystem wurde entwickelt und Rohdaten geordnet, diese den Kategorien zugeteilt und Themen interpretiert. Die Erstellung der Oberkategorien erfolgte deduktiv anhand des Interviewleitfadens, die Entwicklung der Unterkategorien induktiv im Auswertungsprozess. Das Transkript wurde unter Verwendung der Software MAXQDA Analytics Pro 2020 (VERBI GmbH, Berlin; [9]) durch den Erstautor kodiert und die Kodierung durch das Studienteam geprüft. Nach den Vorgaben von Mayring [17] wurde das komprimierte Datenmateri- 
Präv Gesundheitsf 2022 · 17:32-43 https://doi.org/10.1007/s11553-021-00842-z

(c) Der/die Autor(en) 2021

\section{F. A. Neumann · N. Mojtahedzadeh • V. Harth · S. Mache · M. Augustin · B.-C. Zyriax}

\section{Gesundheitsverhalten und -förderung von ambulanten Pflegekräften. Eine explorative, qualitative Studie zur aktuellen Situation, potenziellen Hindernissen, Herausforderungen und Lösungsstrategien aus der Sicht von Expert*innen}

\section{Zusammenfassung}

Hintergrund. Prognosen zufolge werden im Jahr 2050 bis zu 0,85 Mio. Fachkräfte in der Pflege fehlen. Bereits heute führen zunehmende Arbeitsbelastungen zu hohen Krankenständen bei den Beschäftigten. Wissenschaftliche Erkenntnisse zum Gesundheitsverhalten bei ambulanten Pflegekräften fehlen, um zielgruppenspezifische Maßnahmen der betrieblichen Gesundheitsförderung (BGF) bereitstellen zu können.

Ziel der Arbeit. Ziel ist es, das Gesundheitsverhalten von ambulanten Pflegekräften sowie bestehende Angebote der BGF in der ambulanten Pflege zu charakterisieren und Erfahrungen der Expert*innen bei der Entwicklung und Implementierung von Maßnahmen BGF zu erfassen.
Material und Methoden. Zehn Expert*innen aus der Hamburger Pflegebranche und -wissenschaft wurden im Rahmen eines Fokusgruppeninterviews im Januar 2020 befragt. Die Datenauswertung fand mittels qualitativer Inhaltsanalyse statt.

Ergebnisse. Um selbstbestimmt und selbstständig mit der eigenen Gesundheit umzugehen, betonten die Experten, sei es besonders wichtig, die Pflegekräfte für die Themen Ernährung, körperliche Aktivität und Rauchen zu sensibilisieren. Für Stressbewältigung standen hingegen vermehrt verhältnispräventive Maßnahmen, wie realistische Fahrtenplanung, Arbeitszeitplanung und Anpassung der Pausenzeiten, im Fokus.
Schlussfolgerung. Die Arbeitsbedingungen der ambulanten Pflege wirken sich nachteilig auf das Ernährungs- und Trinkverhalten sowie die körperliche Aktivität bei den Beschäftigten aus. Weitere quantitative Erhebungen zum Gesundheitsverhalten werden benötigt, um die gewonnenen Einschätzungen der Expert*innen zu stärken. Zielgruppenspezifische Maßnahmen der BGF müssen an die ambulante Pflege angepasst sein und sowohl verhaltens-, als auch verhältnispräventive Ansätze einbeziehen.

Schlüsselwörter

Ambulante Pflege - Arbeitsmedizin . Arbeitseinfluss · Arbeitsbelastung · Lebensstil

Health behaviour and health promotion of outpatient caregivers. an exploratory, qualitative study on the current situation, potential obstacles, challenges and solution strategies from the perspective of experts

\begin{abstract}
Background. According to forecasts, there will be a shortage of up to 0.85 million skilled workers in healthcare by 2050. Increasing workloads are already leading to high levels of sick leave among employees. There is a lack of scientific knowledge about the health behaviour of outpatient caregivers to be able to develop and provide target group-specific measures for workplace health promotion (WHP).

Objectives. Based on the opinions of experts, the aim was to characterise the health behaviour of outpatient caregivers and to determine existing WHP measures in outpatient care. In addition, experts experiences with regard to the development
\end{abstract}

and implementation of WHP measures were recorded.

Materials and methods. Ten experts from the Hamburg care industry and science were surveyed in a focus group interview in January 2020. The data were evaluated using qualitative content analysis.

Results. As a basis for self-determination and independence in dealing with one's own health, the experts on nutrition, physical activity and smoking emphasised the central importance of awareness-raising measures for nursing staff. To cope with stress, the focus was increasingly on preventive measures such as realistic trip planning, working time planning and adjusting break times.
Conclusions. The working conditions of outpatient care have a negative effect on the eating and drinking behaviour as well as the physical activity of the employees. Quantitative surveys on the health behaviour of outpatient nurses are required in order to strengthen the experts' assessments. Target group-specific measures of WHP must be adapted to outpatient care and include both behavioural and preventive approaches.

Keywords

Ambulatory care - Occupational health - Work influence - Occupational stress · Healthy lifestyle al des Kodierungssystems abschließend in einem separaten Dokument zusammengefasst. Zur Stärkung der Validität fand im Zuge der Analyse eine nähere Betrachtung des möglichen Einflusses des Studienteams auf die Datenerfassung und -auswertung statt und alternative Interpretationswege für als kritisch bewertete Aussagen wurden diskutiert. Die Studienergebnisse sind den Forschungs- fragen und dem entwickelten Kodierungssystem (Anhang, - Tab. 3) folgend dargestellt. Bei direkten Zitaten wurde markiert, von welcher Expertengruppe die Aussage stammt (Berufsverband [BV]; ambulante Pflegedienstleitung [AP]). Zur Beschreibung der Studie wurde die COREQ(„COnsolidated criteria for REporting Qualitative research“)Checkliste verwendet [26].

\section{Ergebnisse}

\section{Gesundheitsverhalten}

In den Kategorien „körperliche Aktivität“, „Ernährungs- und Trinkverhalten“, „Rauchen“ und „Stressbelastung“ finden Aussagen der Expert*innen nähere Betrachtung, in welchen das Gesundheitsverhalten der Beschäftigten eingeschätzt 
und der Einfluss der Arbeitsbedingungen auf das Gesundheitsverhalten beschrieben wurde.

\section{Körperliche Aktivität}

Die Expert ${ }^{*}$ innen beschrieben, dass sich ambulante Pflegekräfte bereits im Berufsalltag viel bewegen. Auf körperliche Aktivität in der Freizeit wurde hingegen nicht näher eingegangen. Die Expert*innen setzten stattdessen voraus, dass die körperliche Aktivität von ambulanten Pflegekräften in der Freizeit unzureichend ist und verwiesen darauf, dass sich die Arbeitsbedingungen hemmend auf die Ausübung körperlicher Aktivität auswirken. Unter „körperliche Aktivität" ergab sich infolgedessen die Kategorie „bewegungshemmende Faktoren“.

Die Beiträge zu den bewegungshemmenden Faktoren wurden den Themen „Erschöpfung erhöht das Bedürfnis nach Regeneration“, „unregelmäßige zeitliche Verfügbarkeit zur Ausübung von Sportroutinen“ und „motivationshemmende Arbeitsbedingungen“ zugeordnet. Infolge von Arbeitsbelastungen gaben die Expert ${ }^{*}$ innen Erschöpfung als Ursache dafür an, dass ambulante Pflegekräfte wenig körperliche Aktivität in der Freizeit betreiben. Hierbei bewirken sowohl die erlebte Stressbelastung als auch die körperliche Erschöpfung durch den Arbeitsalltag, dass in der Freizeit das Bedürfnis nach Regeneration gesteigert wird:

BV: Ein Alltag in der Pflege ist grundsätzlich sehr stressbehaftet. Man [...] bewegt sich im Alltag schon sehr viel. Man kommt nach Hause, ist müde und ist froh, einfach mal die Füße hochzulegen und nichts $z u$ machen.

Eine Verbandsvertretung schilderte, dass der Pflegebetrieb die zeitgebundene Erfüllung der Pflegeleistung sicherstellen muss, wodurch nicht gewährleistet werden kann, dass die Beschäftigten regelmäßig an denselben Wochentagen frei bekommen. Die Initiierung und Einhaltung sportlicher Routinen ist erschwert und die Pflegekraft in ihrer Wahl der Sportart limitiert, da regelmäßige Kurs- und Trainingszeiten nur bedingt eingehalten werden können:

BV: Ich kann sehr selten Teamsport machen. [...], wenn es da keine Flexibilität in den Dienstplänen gibt, und ich [nicht] sagen kann [...]: Mittwochabend möchte ich definitiv keinen Spätdienst machen, weil ich da [...] Handball [...] habe.

Weiterhin wurde festgestellt, dass ambulante Pflegekräfte somit vermehrt auf zeitunabhängige Individualsportarten angewiesen sind. Eine Pflegedienstleitung schlussfolgerte, dass die Erfüllung extrinsischer Grundmotive, wie der Wunsch nach Zugehörigkeit zu einer Sportgruppe, einerseits durch die unregelmäßige zeitliche Verfügbarkeit erschwert ist. Andererseits stehen der intrinsischen Motivation zur Durchführung von Individualsportarten die Erschöpfung in Folge von Arbeitsbelastungen sowie der Wunsch nach Regeneration gegenüber.

\section{Ernährungs- und Trinkverhalten}

Die Aussagen zum Ernährungs- und Trinkverhalten ließen sich in die Kategorien „Einschätzung über das Ernährungs- und Trinkverhaltens " und „Arbeitseinflüsse auf das Ernährungsund Trinkverhalten" unterteilen.

Die Expert*innen beschrieben das Ernährungsverhalten ambulanter Pflegekräfte als unterschiedlich, eine Bewertung der Ernährungsqualität erfolgte nicht. Das Trinkverhalten hingegen wurde als „eher schlecht“ beurteilt. Trinkmengen wurden insgesamt als zu gering eingeschätzt. Der Konsum koffeinhaltiger Getränke galt als favorisiert. Als berufliche Faktoren, welche das Ernährungs- und Trinkverhalten ambulanter Pflegekräfte beeinflussen, hoben die Expert*innen das mobile Setting und die Patienten hervor. Laut den Expert*innen werden unzureichende Trinkmengen bei den Beschäftigten durch die limitierte Verfügbarkeit von Toiletten unterwegs begünstigt:

AP: [...] ich weiß auch, dass Pflegekräfte sagen, sie können nicht so viel trinken. Sie wissen nicht, wo sie auf Toilette gehen können. Natürlich können sie reinkom- men ins Büro und so weiter. Aber das machen die nicht. Also trinken sie eher nichts.

Hinzu wies die Leitung eines ambulanten Pflegedienstes explizit darauf hin, dass die Pausenzeiten und Räumlichkeiten der Pflegebetriebe von den Beschäftigten zur Mahlzeiteneinnahme genutzt werden. Einige Beschäftigte nehmen ihre Mahlzeiten dennoch im Auto bei der Fahrt zu sich:

AP: [...] es gibt Pausenzeiten. Das heißt, die Büros sind mittags gefüllt mit Mitarbeitern, die dort ihre Mahlzeiten zu sich nehmen. Klar gibt es welche, die [...] unterwegs im Fahren dann etwas essen [...].

Ergänzend wurde festgestellt, dass bei der Einnahme von Mahlzeiten im Auto häufig Snacks und Süßigkeiten verzehrt werden. Eine Pflegeleitung merkte an, dass es als Zeichen der Dankbarkeit eine übliche Geste von Patienten und deren Angehörigen sei, Süßigkeiten an die Pflegekräfte zu verschenken. Die Bereitstellung ungesunder Lebensmittel fördere deren Verzehr durch die Beschäftigten. Auf die Geschenke der Patienten Einfluss zu nehmen, erachteten die Expert ${ }^{\star}$ innen als komplex:

AP: Und dann haben wir diese klassische Phase, Zustand nach Frühdienst, [...], völlig ausgehungert und nun liegt die [Schokolade] da. [...] wir können [...] unseren Kunden nicht sagen, wir möchten gerne [...] Nüsschen statt Schokolade. [lachen]

\section{Rauchen}

Es wurde deutlich gemacht, dass sich das Rauchverhalten innerhalb der Pflegebetriebe in den vergangenen Jahrzehnten bereits verbessert hat:

$B V:$ [...] vor zwanzig Jahren, da bin ich in den Pflegedienst reingekommen, da schlug einem [...] eine Wolke entgegen, das ist [...] heutzutage nicht mehr so. [...] in der Mitte war so ein Riesen-Aschenbecher, wo alle schnell [...] geraucht [haben]. [...] ich glaube, das gibt es nicht mehr. 


\section{Stressbelastung}

Die Expert*innen charakterisierten ambulante Pflegekräfte als psychisch stark belastet. Von stressreduzierenden Verhaltensweisen der Beschäftigten wurde nicht berichtet. Die Aussagen zum Thema „Stressbelastung“" wurden der Kategorie „stressfördernde Faktoren“ zugeordnet.

Für „stressfördernde Faktoren“ wurden „Termin- und Leistungsdruck“, „Überforderung“ und „emotionale Bindung zum Patienten“ als Unterkategorien ermittelt. Eine Verbandsvertretung verwies darauf, dass Termin- und Leistungsdruck als Belastungsfaktoren für ambulante Pflegekräfte gelten. Dennoch sind Pflegebetriebe als Wirtschaftsunternehmen auf die Produktivität der Beschäftigten angewiesen, sodass der Arbeitgebende bei der Tourendisposition zwischen der Profitabilität für den Betrieb und der Zumutbarkeit für die Beschäftigten abwägen muss. In der Praxis gelingt dies nicht immer, wodurch ambulante Pflegekräfte unter Termindruck gesetzt werden. In Einzelfällen wird laut einer Verbandsvertretung den Beschäftigten mit unbezahlten Überstunden gedroht:

BV: [...] bei der Tourendisposition fängt das Ganze ja an. [...] Ich habe das von Pflegediensten gehört, die sagen: „Ja, da musst du aber beim Nächsten immer in zehn Minuten sein. Und wenn du das nicht schaffst, [...] dann wird es dir von der Zeit abgezogen. “[...] (AP: „Ich wüsste jetzt einen. ") [...] man kriegt da manchmal so Absonderlichkeiten mit, das kann nicht wahr sein.

Ein erhöhtes Verkehrsaufkommen stehe laut einer Pflegeleitung zudem der Erwartungshaltung der Patienten gegenüber, dass Pflegekräfte Pünktlichkeit bewahren. Verzögerungen beim Transport und der infolgedessen entstehende zeitliche Engpass können dazu führen, dass die Beschäftigten nicht gewillt sind, Pausenzeiten einzuhalten:

AP: [...] sie machen oft ihre Pause nicht, wollen es gar nicht. [...] wir verpflichten sie eigentlich dazu [...].
Eine Verbandsvertretung ergänzte, dass krankheitsbedingte Ausfälle den Terminund Leistungsdruck erhöhen. Durch Erkrankungen erforderliche Ersatzdienste und Zusatzarbeiten wurden als große Belastung für ambulante Pflegekräfte identifiziert. Da die Patienten versorgt werden müssen, ist es seitens der Arbeitgebenden eine Einstellungsvoraussetzung die Beschäftigten kurzfristig einberufen zu können. Die Gewissheit darüber erschwert es den Pflegekräften in der Freizeit abzuschalten und auch der Eintrittsfall sorge, nach Aussage eines Experten, für Frustration:

$B V: X$ ist krank geworden, hat aber gerade erst Bescheid gesagt. Ja, danke schön, hättest du auch gestern Abend sagen können. [...] und wer macht das jetzt eigentlich? Natürlich macht das jemand. [...] der Job muss gemacht werden. Die Patienten müssen versorgt werden. Das ist immer die Einstellungsvoraussetzung [...], die mentale und auch die vom Arbeitgeber. [...] Das sind viele Faktoren, die da auf einen drücken.

Die Verbandsvertretung argumentierte zudem, dass der Fachkräftemangel in der Pflege hierbei eine zusätzliche Rolle spielt, wodurch fehlendes Personal nicht ersetzt werden kann. Oftmals besteht nur die Möglichkeit Zusatzarbeit auf mehrere Beschäftigte zu verteilen, was wiederum die Terminvergabe mit den Patienten erschwert und für eng getaktete Touren sorgt. In Folge der vielseitigen und stetigen Belastungen stellte eine Verbandsvertretung fest, dass insbesondere Berufseinsteiger in der Pflege leicht von dem Anforderungsprofil ihres Berufs überfordert werden. Bewältigungsstrategien sind den Expert*innen nach im Umgang mit erlebtem Termin- und Leistungsdruck wichtig, um der Überforderung der Beschäftigten vorzubeugen.

BV: Wie kriege ich da überhaupt Entspannung rein [...]? Dass ich sage, ja gut, dann ist das eben zehn Minuten später [...] und ich muss nicht immer in dieser Alarmstimmung unterwegs sein. Hilfe, ein Notfall, ich muss da hin [...]. Und da bin ich heilfroh, wenn dann die Schicht vorbei ist [...].
Eine Pflegedienstleitung der ambulanten Intensivpflege ergänzte, dass auch zwischenmenschliche Aspekte eine Rolle spielen. Insbesondere bei der Eins-zueins-Betreuung kann das Entstehen einer emotionalen Bindung zum Patienten Stressbelastung verursachen:

AP: [...] der Mitarbeiter [ist] vor Ort [...] und kriegt alles mit, was er nicht wissen will. [...] auch umgekehrt gibt er zwangsläufig [...] was von sich Preis. Da ist [...] Distanz-Nähe-Verschiebung eben das gro$\beta e$ Thema und das macht natürlich Stress. Ich hatte jetzt gerade wieder den Fall, dass eine Mitarbeiterin [...] sich den Klienten $z u$ dicht rangeholt hat und dann irgendwann ist es eskaliert und dann war auch die Erde so verbrannt, dass sie da nicht mehr eingesetzt werden konnte.

\section{Betriebliche Gesundheits- förderung}

Aussagen der Expert*innen zur BGF bezogen sich auf bereits verfügbare Maßnahmen sowie wünschenswerte Umsetzungsideen für zukünftige Programme. Alle genannten Maßnahmen der BGF wurden gelistet (Anhang, - Tab. 4). Diese deckten die untersuchten Gesundheitsthemen $\mathrm{ab}$ und wurde den Kategorien „Verhaltensprävention“ (auf das individuelle Verhalten abzielend) und „Verhältnisprävention" (auf gesundheitsförderliche Arbeitsstrukturen abzielend) zugeordnet.

\section{Verhaltensprävention}

Mit individuellen Beratungen, Kursen, betriebsinternen Veranstaltungen zur Teambildung sowie Mentorenprogrammen liegt laut Expert*innen auf der verhaltenspräventiven Ebene ein vielseitiges Angebot an Maßnahmen für BGF vor. Seminare zur gesunden Ernährung, individuelle Ernährungsberatungen, Raucherseminare sowie Kurse zu Stressbewältigung und Resilienz werden bereitgestellt. Weiterhin verfügen viele Pflegedienste über Kooperationen mit Fitnessstudios und Sportvereinen, wodurch eine vergünstigte Teilnahme an Sportprogrammen ermöglicht wird. Eine gesundheitsförderliche Unternehmenskultur soll gefördert werden. Eine 
Pflegedienstleitung beschrieb, dass wöchentlich ein gemeinsames Frühstück angeboten und ein Obstkorb mit frischem, kostenlosem Obst zur Verfügung gestellt wird. Eine Gummibärchenschale, welche in der Vergangenheit am Empfang stand, wurde durch Weintrauben ersetzt und den Beschäftigten werden Supplemente angeboten:

AP: Wir kaufen dann noch Vitamin C. Also nicht nur Obst, sondern Vitamin $C$ in Tees oder in Pulverform [...]. Wer mag, kann das nehmen. [...] ernährt euch gesund [...] und tu was Gesundes für dich und uns ist es wichtig.

Weiterhin erhalten Beschäftigte, welche die Strecken zwischen den Patienten mit dem Fahrrad zurücklegen möchten, eine Fahrradausrüstung. Eine Pflegedienstleitung erläuterte, dass in den Diensträumen des Pflegediensts zeitweise wöchentlich Massagen sowie Kurse zu Yoga und aktiver Rückenschule angeboten wurden. Veranstaltungen zur Teambildung, wie gemeinsame Sportgruppen und Fahrradtouren, werden vom Betrieb organisiert. Um Berufseinsteigern im Umgang mit den vielseitigen Arbeitsbelastungen $\mathrm{zu}$ unterstützen, werden erfahrene Pflegekräfte betriebsintern als Mentoren zur Seite gestellt.

\section{Verhältnisprävention}

Den Einbezug verhältnispräventiver Maßnahmen erachteten die Expert*innen ebenfalls als wichtig und fokussierten hierbei verstärkt auf die Dienstplangestaltung. Eine Pflegedienstleitung berichtete, dass zur Gewährleistung von freien Tagen, Dienstpläne mit Rufbereitschaft und Schichten an maximal 5 aufeinanderfolgenden Arbeitstagen je Beschäftigten eingeführt wurden. Sofern möglich, sollte ein Mitspracherecht bei der Dienstplangestaltung eingeräumt werden:

AP: $[. .$.$] da ist halt das einzige Steue-$ rungsinstrument wirklich, den Mitarbeitern einen gewissen Freiraum bei der Dienstplangestaltung mit einzuräumen, [...] die müssen da [...] ein Mitspracherecht haben. Und möglichst [...] Festwünsche, $[. .$.$] die [. .$.$] immer bestehen [...],$ sonst hast du irgendwann die Krankmeldung auf dem Tisch.

Auch Reglementierungen, wie ein Rauchverbot im Auto, finden als gesundheitsfördernde Rahmenbedingung Anwendung.

\section{Hindernisse und Heraus- forderungen betrieblicher Gesundheitsförderung}

Folgend werden Anmerkungen der Expert*innen $\mathrm{zu}$ berufsgruppenspezifischen Besonderheiten dargestellt, welche sich bei der Entwicklung und Implementierung von Maßnahmen der BGF potenziell als Hindernisse und Herausforderungen auswirken können. Die Aussagen der Expert*innen konnten den Kategorien „hinderliche Arbeitsbedingungen“, „Perspektive der Arbeitgebenden“ und „Einflussfaktoren auf die Teilnahmebereitschaft der Beschäftigten“ zugeordnet werden.

\section{Hinderliche Arbeitsbedingungen}

Die Aussagen zu den hinderlichen Arbeitsbedingungen ließen sich in die Kategorien „Arbeitszeiten und -plätze“ und „arbeitsbezogene Vorteile durch schlechtes Gesundheitsverhalten “ unterteilen. Eine grundlegende Herausforderung für BGF in der ambulanten Pflege sind laut Expert*innen die unterschiedlichen Arbeitszeiten im Schichtsystem und die Arbeit außerhalb des Betriebs. Es ist schwierig, Angebote so zu gestalten, sodass diese für alle Beschäftigten gleichermaßen nutzbar sind. Weiterhin kann ein nachteilhaftes Gesundheitsverhalten die Arbeitsbedingungen vorteilhaft beeinflussen. Eine Pflegedienstleitung merkte an, dass ambulante Pflegekräfte zeitliche Entlastungen durch das Auslassen von gesetzlich vorgeschriebenen Pausen schaffen. Regelmäßiges Rauchen wiederum kann einer Verbandsvertretung zu Folge als Vorwand zur Gewinnung zusätzlicher Pausenzeiten angewendet werden.

\section{Perspektive der Arbeitgebenden}

Um die erfolgreiche Implementierung von Maßnahmen der BGF zu gewährleisten, ist die Unterstützung seitens der Arbeitgebenden notwendig. Eine Pflegedienstleitung verwies darauf, dass Arbeitgebende grundsätzlich eher positiv gegenüber BGF eingestellt sind, da sich durch Gesundheitsförderung eine Verringerung der Krankentage erhofft wird. Nichtsdestotrotz wurden unter den Kategorien „Wirtschaftlichkeit“ und „Verantwortung für Gesundheit“ Perspektiven identifiziert, welche bei der BGF-Umsetzung Hindernisse darstellen können. Eine Verbandsvertretung verdeutlichte, dass der Pflegebetrieb als Wirtschaftsunternehmen finanzielle und leistungsorientierte Interessen vertritt:

$B V$ : Ich versuche [...], dass ich meine Dienste vollkriege, dass ich [...] das mit meinem begrenzt vorhandenen Pflegepersonal auf die Reihe bekomme. Dann kann es sehr schnell dazu kommen, dass [...] das Gesundheitsverhalten [... ] eine Randerscheinung ist für mich, [...] die Gesundheit meiner Pflegekräfte [...] nicht erste Priorität der Leitungsebene ist, definitiv nicht.

Daraus resultiert, dass Maßnahmen der BGF meist auf freiwilliger Basis in der Freizeit angeboten werden. Außerdem wird seitens des Arbeitgebenden die Verantwortung für den Lebensstil bei den Beschäftigten selbst gesehen:

AP: Ich kann Bereitschaft schaffen, aber ich kann die [Beschäftigten] nicht umerziehen. Wenn sich jemand von Cola und Pommes ernähren will, dann kann ich das auch nicht ändern. Will ich auch nicht.

Bei Belastungen, welche wie Stress und Rückenbeschwerden auf den Arbeitsbedingungen beruhen, bekam eine Verbandsvertretung von Pflegekräften zurückgespiegelt, dass dort die Verantwortung beim Arbeitgebenden liegt:

$B V:[\ldots]$, dass man da gar nicht [...] mit „Wir machen einen Kurs Stressmanagement" [...] komm[t], weil [...] die Pflegekräfte [...] in Interviews gesagt haben: „Die Verantwortung liegt da wieder bei mir, aber eigentlich ist es Aufgabe des Arbeitgebers, mir [...] Verhältnisse $z u$ schaffen, dass ich eben nicht diese Stressbelastung habe." 


\section{Einflussfaktoren auf die Teilnahmebereitschaft der Beschäftigten}

Weitere Einflussfaktoren, welche die Teilnahmebereitschaft der Beschäftigten hemmen, sammelten sich in den Themengebieten „Bewusstsein und Motivation für $\mathrm{BGF}^{\text {“ }}$ und „Heterogenität der Berufsgruppe“.

Den Expert*innen zufolge fehlt vielen Beschäftigten das Bewusstsein, dass Gesundheit und demzufolge Gesundheitsförderung für ein langes Berufsleben wichtig sind. Vergangene und aktuelle Angebote wurden nur von jenen Pflegekräften angenommen, welche „von sich aus schon ein eigenes Bewusstsein dafür (Wichtigkeit von Gesundheit) haben“. $\mathrm{Da}$ es den Beschäftigten obliegt, ob BGFMaßnahmen in der Freizeit genutzt werden, präferieren jene ohne Bewusstsein die Erfüllung anderer Bedürfnisse:

BV: Wenn dann die Frage kommt: „Nutzen Sie das auch?" [...] - „Nein, wenn ich nach Hause komme, lege ich doch lieber schnell die Füße hoch, als dass ich noch irgendwo ins Fitnessstudio gehe“.

Laut Expert*innen bedarf es zusätzlichen Anreizen zur Steigerung der Motivation. Damit BGF nicht als Zusatzbelastung wahrgenommen wird, stellt die Verpflichtung zur Teilnahme keine zielführende Option dar. Pflegekräfte sind verschiedener Herkunft, Ethnien und Altersklassen und unterscheiden sich in ihren Wünschen und Erwartungen hinsichtlich BGF, wodurch eine zielgruppenspezifische Gestaltung der Maßnahmen erschwert ist. Weiterhin berichteten die Expert ${ }^{*}$ innen, sowohl durch kostenlose Kursangebote als auch bei der Auszahlung von Teilnahmeboni bereits auf Verwunderung seitens Beschäftigter mit Migrationshintergrund gestoßen zu sein. Die Wahrnehmung darüber, inwiefern der Arbeitgebende für die Gesundheit der Beschäftigten verantwortlich ist, scheint sich ebenfalls vor dem kulturellen Hintergrund $\mathrm{zu}$ unterscheiden.

\section{Lösungsstrategien}

Die Aussagen zu Lösungsstrategien im Umgang mit Hindernissen und Herausforderungen resultierten in den Katego- rien: „Pflegedienstleitungen als Erfolgsfaktoren“, „Motivation schaffen“, „Partizipation bei der Entwicklung von Maßnahmen“ und „E-Health“.

Pflegedienstleitungen nehmen eine Vorbildfunktion ein und stellen somit einen wichtigen Faktor für die erfolgreiche Implementierung von BGF dar:

AP: Wenn die Leitung es macht, zeigt es, dass es wichtig ist. [...] Und ich glaube, dass [...] gerade auf die jüngeren Kollegen, die hinten nachkommen, die müssen das gleich [...] sehen, dass es [...] eine Rolle spielt.

Somit ist es notwendig, eng mit den Leitungsebenen der Pflegebetriebe zusammenzuarbeiten. Eine gemeinsame systematische Betrachtung der krankheitsverursachenden Belastungsfaktoren kann zudem das Bewusstsein der Führungskräfte darüber stärken, welche Veränderungen im Betriebsablauf die Gesunderhaltung positiv beeinflussen können. Weiterhin können laut den Expert*innen die wiederholte Initiierung zur Teilnahme durch die Pflegedienstleitungen, die Belegung von Weiterbildungskursen mit Arbeitszeit und zielgruppenspezifische Incentives bei den Beschäftigten als extrinsische Motivatoren fungieren. Nichtsdestotrotz sei es empfehlenswert, die Pflegekräfte in die Entwicklung der BGF-Maßnahmen einzubeziehen. Eine Pflegedienstleitung argumentierte, dass der persönliche Lebensstil eine autonome Entscheidung ist. Um die Teilnahmebereitschaft der Pflegekräfte an der BGF zu steigern, könnte ein partizipativer Ansatz bei der Entwicklung von Maßnahmen förderlich sein. Die Expert*innen befürworteten, die Themen Digitalisierung und E-Health tiefergehend zu betrachten. Für das mobile Setting der ambulanten Pflege stelle Gesundheitsförderung im Rahmen von E-Health eine naheliegende Option dar. Um ältere, wenig technikaffine Pflegekräfte zu erreichen, wären jedoch andere Maßnahmen notwendig.

\section{Diskussion}

Nach unserem Kenntnisstand ist dies bislang die erste Studie, welche Gesundheitsverhalten und -förderung sowie
Hindernisse, Herausforderungen und Lösungsstrategien für die Entwicklung und Implementierung von Maßnahmen der BGF für ambulante Pflegekräfte explorativ untersucht. In der Fokusgruppe mit Expert*innen aus der Pflege wurde in Hinsicht auf das Gesundheitsverhalten der Beschäftigten explizit in den Bereichen Ernährungs- und Trinkverhalten, körperliche Aktivität und Stressbelastung weitestgehend von arbeitsbedingten Einschränkungen berichtet. Die hohen körperlichen und mentalen Belastungen der Pflegekräfte sowie die finanziellen und leistungsorientierten Interessen der Pflegebetriebe als Wirtschaftsunternehmen stehen der BGF als Hindernis gegenüber. Zur erfolgreichen Umsetzung von Maßnahmen der BGF können Pflegedienstleitungen in ihrer Rolle als Vorbildfunktion sowie extrinsische Anreize eine Schlüsselrolle einnehmen.

\section{Die Arbeitsbedingungen in der ambulanten Pflege können sich negativ auf das Gesundheitsverhalten der Beschäftigten auswirken}

Durch die Expert*innen erfolgte zumeist keine direkte Beurteilung des Gesundheitsverhaltens ambulanter Pflegekräfte, nichtsdestotrotz verdeutlichten deren Aussagen, dass die Aufrechterhaltung gesundheitsförderlicher Verhaltensweisen für ambulante Pflegekräfte durch die Arbeitsbedingungen erschwert ist. Beispielsweise sind die Beschäftigten aufgrund der limitierten Verfügbarkeit von Toiletten unterwegs in ihrer Flüssigkeitszufuhr gehemmt. Trinkmengen werden während der Arbeitszeit reduziert, da die Rückkehr in den Pflegedienst zur Verrichtung der Notdurft gleichbedeutend mit Zeitverlust ist. Beispiele für solch zeitersparende Bewältigungsverhalten, wie das bewusste Auslassen von Pausen und die Einnahme von Mahlzeiten im Auto [16], scheinen bei ambulanten Pflegekräften vermehrt aufzutreten. Im beruflichen Umfeld haben Terminund Leistungsdruck somit wesentlichen Einfluss auf das Gesundheitsverhalten ambulanter Pflegekräfte [13]. Bei stationären Pflegekräften wurde die Assoziation von Termin- und Leistungsdruck 
mit dem Burnout-Syndrom dokumentiert [6]. Wirth et al. [29] ermittelten zum Gesundheitsstatus und -verhalten von Auszubildenden in der Alten- und Krankenpflege, dass die Prävalenz von Übergewicht und Rauchen im Vergleich zum Durchschnitt der deutschen Bevölkerung erhöht war. Weiterhin gaben $73,1 \%$ der Befragten Altenpfleger an, in der Woche weniger als $2 \mathrm{~h}$ körperliche Aktivität zu betreiben, 40,8\% gingen ungesunden Ernährungsweisen nach und weitere $40,8 \%$ verfolgten einen gefährlichen Alkoholkonsum. Die Schlussfolgerungen der Expert ${ }^{*}$ innen für ambulante Pflegekräfte basieren teilweise auf vergleichbaren Annahmen. Um diese zu bestätigen, detailliertere Erkenntnisse zu generieren sowie neue Ansatzpunkte für die BGF zu identifizieren, werden zukünftig systematische quantitative Basiserhebungen zum Gesundheitsverhalten benötigt. Zudem sollten die Gesundheitskompetenzen untersucht werden, da diese einen maßgeblichen Einfluss auf das Gesundheitsverhalten ambulanter Pflegekräfte haben können [11]. Es bleibt festzuhalten, dass der Bedarf an BGF durch die Expert ${ }^{*}$ innen verdeutlicht wurde.

\section{Die körperlichen und mentalen Arbeitsbelastungen limitieren die Bereitschaft der ambulanten Pflegekräfte für die Teilnahme an BGF}

Während der Fokusgruppe wurde eine Vielzahl verhaltens- und verhältnispräventiver Maßnahmen BGF in der ambulanten Pflege genannt (Anhang, - Tab. 4). Gleichwohl blieben laut der Expert ${ }^{\star}$ innen die Angebote in der Vergangenheit zu häufig ungenutzt. Neben hinderlichen Berufsbedingungen für die erfolgreiche BGF-Umsetzung, wie variierende Schichtzeiten und das mobile Arbeitssetting, wurde v. a. die mangelnde Teilnahmebereitschaft der ambulanten Pflegekräfte als Herausforderung hervorgehoben. Geringe Teilnahmequoten an Maßnahmen zur Förderung der körperlichen Aktivität wurden auf die limitierte Gewissheit bei der Freizeitplanung und mangelnde Motivation infolge der Erschöpfung vom Arbeitstag zurück- geführt. Letzteres geht mit Ergebnissen von Zeiher et al. [30] einher, welche bei weiblichen Beschäftigten eine starke Assoziation zwischen Freizeitaktivität und beruflicher körperlicher Aktivität nachwiesen. Frauen, welche in ihrer Freizeit keine körperliche Aktivität betrieben, hatten zugleich eine schlechte kardiorespiratorische Fitness, insbesondere wenn sie in einem körperlich anstrengenden Berufsfeld wie der Pflege arbeiteten.

\section{Für nachhaltige BGF müssen ver-} haltens- und verhältnispräventive Maßnahmen sowohl zielgruppenals auch branchenspezifisch sein

Das Beispiel zur Teilnahmebereitschaft verdeutlicht, dass in der ambulanten Pflege verhaltenspräventive Maßnahmen allein nicht ausreichen. Es werden ganzheitliche Programme der BGF benötigt, welche sowohl auf das Verhalten der Beschäftigten, als auch auf die Verhältnisse, wie Arbeitsbedingungen und -organisation, abzielen [19]. In der Vergangenheit wurde gezeigt, dass so, neben der Risikoreduzierung für gesundheitsgefährdende Beanspruchungsfolgen, auch die Produktivität und Anwesenheit der Beschäftigten verbessert werden können [22, 24]. Die Verantwortung für die Gesundheit der ambulanten Pflegekräfte wird je nach Gesundheitsthema unterschiedlich verortet. Da der arbeitsbezogene Stress primär von den Arbeitsbedingungen abhängt [13], werden die Betriebe von den Beschäftigten in der Handlungsverantwortung gesehen. Insbesondere durch Optimierung und Anpassung von Schicht-, Touren- und Pausenzeitplanung kann verhältnispräventiv für Entlastung gesorgt werden [19]. Als Basis für Selbstbestimmtheit und Selbstständigkeit im Umgang mit der eigenen Gesundheit, verorteten die Expert*innen die Verantwortung für Ernährung, körperliche Aktivität und Rauchen bei den Beschäftigten. Nichtsdestotrotz sind die Betriebe neben der Bereitstellung gesundheitsförderlicher Arbeitsbedingungen auch dazu verpflichtet, einen gesundheitsgerechten Lebensstil der Beschäftigten zu unterstützen [7]. Einhergehend mit dem Arbeitsschutzgesetz zur Umsetzung be- trieblicher Gesundheitsförderung sollten Maßnahmen bedarfsbezogen und unabhängig vom arbeitsrechtlichen Status offenstehen, um auch Beschäftigte mit sozialer Benachteiligung und geringeren Gesundheitschancen anzusprechen. Bei der Bereitstellung von Maßnahmen muss zudem vermehrt auf die Wünsche der heterogenen Berufsgruppe der Pflegekräfte eingegangen und identifiziert werden, wie die Motivation zur Teilnahme gestärkt werden kann. Um frühzeitig das Bewusstsein für die Notwendigkeit von BGF zu wecken und Nachhaltigkeit zu schaffen ist es empfehlenswert, gesundheitsförderliche Rahmenbedingungen und Grundlagen der BGF bereits in der Pflegeausbildung einzuführen und $\mathrm{zu}$ etablieren. Insbesondere für technikaffine Berufseinsteiger könnten die Digitalisierung von Arbeitsabläufen und E-Health-gestützte Gesundheitsförderung zukünftig Lösungen für das mobile Setting der ambulanten Pflege bieten [19]. Die Entwicklung solch innovativer Interventionsprogramme mittels partizipativen Ansatzes stellt sicher, dass BGF zielgruppenspezifisch ist und infolgedessen für eine erhöhte Teilnahmebereitschaft sorgt [1]. Das Mitwirken an der Verbesserung bestehender Angebote und Entwicklung neuer Maßnahmen beherbergt zudem das Potenzial einer verbesserten Einbettung von Angeboten in den Berufsalltag.

\section{Stärken und Limitationen}

Eine Stärke dieser Studie ist es, dass die Sicht von Expert*innen der Pflegewissenschaft, Wirtschaft und Berufsverbänden in einer heterogenen Stichprobe (Alter, Arbeitserfahrung etc.) abgefragt wurde. Die unterschiedlichen Perspektiven der Teilnehmer wurden festgehalten und Themen von Interesse identifiziert. Für das explorative Vorhaben neue Erkenntnisse zu generieren, erwies sich die Erhebungsmethode als geeignet. Transkription nach Dresing und Pehl [8] und die Auswertung gemäß qualitativer Inhaltsanalyse nach Mayring [17] fanden unter strenger Anwendung wissenschaftlicher Richtlinien statt. Die Internationale COREQ-Checkliste [26] wurde zur Erhöhung der internen Validität verwendet, 
die Ergebnisse mit Zitaten aus der Fokusgruppe dargestellt und wo möglich mit empirischer Evidenz belegt.

Eine der größten Stärken und Limitationen zugleich ist es, dass keine Beschäftigten unterhalb einer Leitungsposition für die Fokusgruppe berücksichtigt wurden. Die Zusammensetzung aus Expert*innen erlaubte es insbesondere Erkenntnisse aus struktureller und betrieblicher Perspektive zu gewinnen, birgt jedoch die Gefahr, dass die Interessen der heterogenen multiethnischen Beschäftigtengruppe geringe Beachtung fanden. Weiterhin kann der Auswahlprozess der Teilnehmer, welcher u. a. auf Empfehlungen stattfand, zu Verzerrungen geführt haben. Alle partizipierenden Expert ${ }^{*}$ innen stammten aus der Hamburger Pflegebranche, die Ergebnisse könnten sich somit für andere Städte und v.a. ländlichere Regionen Deutschlands unterscheiden. Es besteht die Möglichkeit, dass das Antwortverhalten der Befragten aufgrund sozialer Erwünschtheit und mangelnder Anonymität zu Mitbefragten sowie Interviewer beeinflusst wurden. Vor dem Hintergrund der demografischen Entwicklung und des anstehenden Fachkräftemangels entsteht zudem ein zeitlicher Kontext. Mit dem Ziel Beschäftigte langfristig zu binden, kann sich seitens der Arbeitgebenden in der Pflege ebenfalls die Bereitschaft verändert haben, verbesserte Arbeitsbedingungen sowie Maßnahmen betrieblicher Gesundheitsförderung anzubieten.

\section{Fazit für die Praxis}

\section{- Zur Reduzierung der Stressbelastung ambulanter Pflegekräfte sollten verhältnispräventive Maßnahmen, wie die Optimierung von Schicht-, Touren- und Pausenzeitplanung herangezogen werden. \\ - Damit verhaltenspräventive Ange- bote frequentierter genutzt werden, müssen die Beschäftigten für die Notwendigkeit von betrieblicher Gesundheitsförderung (BGF) für ihre Gesundheit sensibilisiert werden. Auch der partizipative Einbezug der ambulanten Pflegekräfte in die fort- laufende Optimierung bestehender}

Angebote und Entwicklung neuer Maßnahmen der BGF kann hierbei unterstützen.

- Um die aktuelle Evidenz zu stärken und zusätzliche Ansatzpunkte für die BGF in der ambulanten Pflege zu identifizieren, braucht es systematische, qualitative und quantitative Basiserhebungen zu den Gesundheitskompetenzen und zum Gesundheitsverhalten, insbesondere zu den Themen "Stressbelastung", „Regeneration“, „Ernährungsverhalten", "körperliche Aktivität" und „Rauchen".

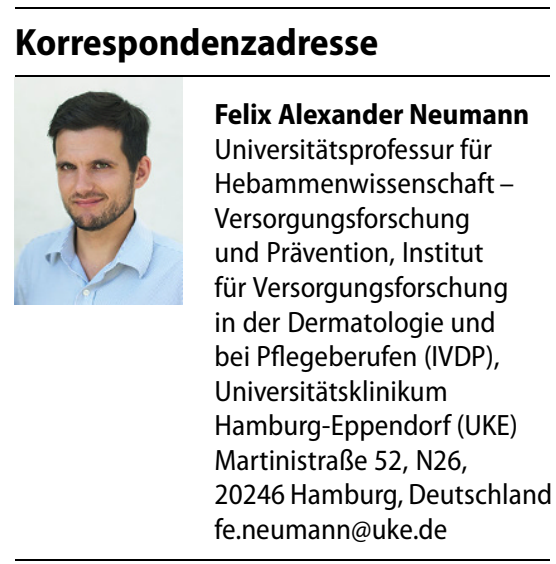

Danksagung. Die Autoren danken dem Scientific Communication Team des IVDP, insbesondere Merle Twesten und Mario Gehoff, für das Lektorat des Artikels.

Förderung. Das Projekt wurde von der Berufsgenossenschaft für Gesundheitsdienst und Wohlfahrtspflege (BGW) gefördert.

Funding. Open Access funding enabled and organized by Projekt DEAL.

\section{Einhaltung ethischer Richtlinien}

Interessenkonflikt. F.A. Neumann, N. Mojtahedzadeh, V. Harth, S. Mache, M. Augustin und B.C. Zyriax geben an, dass kein Interessenkonflikt besteht.

Für diesen Beitrag wurden von den Autoren keine Studien an Menschen oder Tieren durchgeführt. Für die aufgeführten Studien gelten die jeweils dort angegebenen ethischen Richtlinien. 


\section{Anhang}

Tab. 2 Relevante Fragestellungen des Interviewleitfadens

Interviewthema Fragestellungen des Interviews

\begin{tabular}{|c|c|}
\hline \multirow{3}{*}{$\begin{array}{l}\text { Gesundheits- } \\
\text { situation und } \\
\text {-verhalten }\end{array}$} & $\begin{array}{l}\text { I. Wie schätzen Sie die Gesundheitssituation ambulanter Pflegekräfte für die } \\
\text { Gesundheitsthemen (1-4) ein? }\end{array}$ \\
\hline & II. Wird das Gesundheitsverhalten durch die Arbeitsbedingungen beeinflusst? \\
\hline & III. Wenn ja: inwiefern? \\
\hline \multirow{4}{*}{$\begin{array}{l}\text { Maßnahmen } \\
\text { betrieblicher } \\
\text { Gesundheitsför- } \\
\text { derung }\end{array}$} & I. Welche BGF-Maßnahmen sind verfügbar? \\
\hline & II. Wer stellt die BGF-Maßnahmen zur Verfügung? \\
\hline & III. Werden die BGF-Maßnahmen von den Beschäftigten genutzt? \\
\hline & IV. Welche zusätzlichen BGF-Maßnahmen sind wünschenswert? \\
\hline \multirow{2}{*}{$\begin{array}{l}\text { Umsetzung be- } \\
\text { trieblicher Ge- } \\
\text { sundheitsförde- } \\
\text { rung }\end{array}$} & $\begin{array}{l}\text { I. Welche Hindernisse und Herausforderungen sind bei der Entwicklung und } \\
\text { Implementierung von BGF-Maßnahmen zu erwarten? }\end{array}$ \\
\hline & $\begin{array}{l}\text { II. Welche Lösungsstrategien sind für den Umgang mit den Hindernissen emp- } \\
\text { fehlenswert? }\end{array}$ \\
\hline
\end{tabular}

Tab. 3 Kodierungssystem zur Inhaltsanalyse

\begin{tabular}{|c|c|c|}
\hline Thema & $\begin{array}{l}\text { Deduktive } \\
\text { Kategorien }\end{array}$ & Induktive Kategorien \\
\hline \multirow[t]{5}{*}{$\begin{array}{l}\text { Gesundheits- } \\
\text { verhalten }\end{array}$} & $\begin{array}{l}\text { Körperliche } \\
\text { Aktivität }\end{array}$ & $\begin{array}{l}\text { Bewegungshemmende Faktoren: } \\
\text { - Erschöpfung erhöht das Bedürfnis nach Regeneration, } \\
\text { - unregelmäßige zeitliche Verfügbarkeit zur Ausübung von } \\
\text { Sportroutinen, } \\
\text { - motivationshemmende Arbeitsbedingungen }\end{array}$ \\
\hline & \multirow{2}{*}{$\begin{array}{l}\text { Ernährungs- } \\
\text { und Trinkver- } \\
\text { halten }\end{array}$} & Einschätzung über das Ernährungs- und Trinkverhalten \\
\hline & & $\begin{array}{l}\text { Arbeitseinflüsse auf das Ernährungs- und Trinkverhalten: } \\
\text { - Einfluss des mobilen Settings, } \\
\text { - Bereitstellung ungesunder Lebensmittel durch den Patienten }\end{array}$ \\
\hline & Rauchen & Raucherstatus \\
\hline & Stress & $\begin{array}{l}\text { Stressfördernde Faktoren: } \\
\text { - Termin- und Leistungsdruck, } \\
\text { - Überforderung, } \\
\text { - emotionale Bindung zum Patienten }\end{array}$ \\
\hline \multirow{9}{*}{$\begin{array}{l}\text { Betriebliche } \\
\text { Gesund- } \\
\text { heitsförde- } \\
\text { rung }\end{array}$} & \multirow{2}{*}{$\begin{array}{l}\text { Maßnahmen } \\
\text { der BGF }\end{array}$} & Verhaltensprävention \\
\hline & & Verhältnisprävention \\
\hline & \multirow[t]{3}{*}{$\begin{array}{l}\text { Hindernisse } \\
\text { und Heraus- } \\
\text { forderungen }\end{array}$} & $\begin{array}{l}\text { Hinderliche Arbeitsbedingungen: } \\
\text { - Arbeitszeiten und - plätze, } \\
\text { - arbeitsbezogene Vorteile durch schlechtes Gesundheitsverhal- } \\
\text { ten }\end{array}$ \\
\hline & & $\begin{array}{l}\text { Perspektive der Arbeitgebenden: } \\
\text { - Wirtschaftlichkeit, } \\
\text { - Verantwortung für Gesundheit }\end{array}$ \\
\hline & & $\begin{array}{l}\text { Einflussfaktoren auf die Teilnahmebereitschaft der Beschäftigten: } \\
\text { - Bewusstsein und Motivation für BGF, } \\
\text { - Heterogenität der Berufsgruppe }\end{array}$ \\
\hline & \multirow{4}{*}{$\begin{array}{l}\text { Lösungs- } \\
\text { strategien } \\
\text { zum Umgang } \\
\text { mit Heraus- } \\
\text { forderungen }\end{array}$} & Pflegedienstleitungen als Erfolgsfaktoren \\
\hline & & Motivation schaffen \\
\hline & & Partizipation bei der Entwicklung von Maßnahmen \\
\hline & & E-Health \\
\hline
\end{tabular}


Tab. 4 Listung genannter Maßnahmen der Gesundheitsförderung

Körperliche Akti- Kooperationen mit Fitnessstudios und Sportvereinen

vität

Bereitstellung von Fahrradausrüstung

Kursangebote (z. B. Rückenschule) in Pflegedienstnähe oder in den Diensträumen vor Ort

Kurse zur Bewusstseinsbildung/Aufklärung über die Wichtigkeit von körperlicher Aktivität für die Gesundheit

\begin{tabular}{|c|c|}
\hline \multirow{3}{*}{ Rauchen } & Workshops zur Raucherentwöhnung \\
\hline & Rauchverbot im Auto \\
\hline & Anpassung der Pausenzeiten \\
\hline \multirow{8}{*}{$\begin{array}{l}\text { Ernährung und } \\
\text { Trinken }\end{array}$} & Individuelle Ernährungsberatungen \\
\hline & Seminare zur gesunden Ernährung \\
\hline & Gemeinsames Kochevent \\
\hline & $\begin{array}{l}\text { Kurse zur Bewusstseinsbildung/Aufklärung über die Wichtigkeit von Ernäh- } \\
\text { rung für die Gesundheit }\end{array}$ \\
\hline & Bereitstellung von frischem, kostenlosem Obst und Supplementen \\
\hline & Gemeinsames Frühstück \\
\hline & Entwicklung einer "gesunden Unternehmenskultur" \\
\hline & Arrangements für Toiletten außerhalb des Pflegebetriebs \\
\hline \multirow[t]{4}{*}{ Stress } & $\begin{array}{l}\text { Weiterbildungskurse zu Stressbewältigung, Resilienz, Achtsamkeit und fach- } \\
\text { verwandten Themen }\end{array}$ \\
\hline & Mentoren-Programme für Berufseinsteiger \\
\hline & $\begin{array}{l}\text { Optimierung der Zeit-/Schicht-/Tourenplanung } \\
\text { - Vergabe von Termin-Zeitfenstern an Kunden (anstelle von Uhrzeiten) } \\
\text { - Partizipation der Pflegekräfte bei der Dienstplangestaltung } \\
\text { - Dienstplan mit Rufbereitschaften } \\
\text { - Anpassung der Pausenzeiten }\end{array}$ \\
\hline & $\begin{array}{l}\text { Entspannungsangebote } \\
\text { - Yoga } \\
\text { - Massagen (Wellness) }\end{array}$ \\
\hline
\end{tabular}

Open Access. Dieser Artikel wird unter der Creative Commons Namensnennung 4.0 International Lizenz veröffentlicht, welche die Nutzung, Vervielfältigung, Bearbeitung, Verbreitung und Wiedergabe in jeglichem Medium und Format erlaubt, sofern Sie den/die ursprünglichen Autor(en) und die Quelle ordnungsgemäß nennen, einen Link zur Creative Commons Lizenz beifügen und angeben, ob Änderungen vorgenommen wurden.

Die in diesem Artikel enthaltenen Bilder und sonstiges Drittmaterial unterliegen ebenfalls der genannten Creative Commons Lizenz, sofern sich aus der Abbildungslegende nichts anderes ergibt. Sofern das betreffende Material nicht unter der genannten Creative Commons Lizenz steht und die betreffende Handlung nicht nach gesetzlichen Vorschriften erlaubt ist, ist für die oben aufgeführten Weiterverwendungen des $\mathrm{Ma}$ terials die Einwilligung des jeweiligen Rechteinhabers einzuholen.

Weitere Details zur Lizenz entnehmen Sie bitte der Lizenzinformation aufhttp://creativecommons.org/ licenses/by/4.0/deed.de.

\section{Literatur}

1. Andersson N (2018) Participatory research-A modernizing science for primary health care. J Gen Fam Med 19(5):154-159. https://doi.org/10.1002/ jgf2.187
2. Barthelmes I, Bödeker W, Sörensen J, Kleinlercher KM, Odoy J (2019) iga.Report 40. Wirksamkeit und Nutzen arbeitsweltbezogener Gesundheitsförderung und Prävention. iga, Dresden (Zusammenstellung der wissenschaftlichen Evidenz 2012 bis 2018)

3. Braun V, Clarke V (2006) Using thematic analysis in psychology. Qual Res Psychol 3(2):77-101. https:// doi.org/10.1191/1478088706qp063oa

4. Craigie M, Slatyer S, Hegney D, Osseiran-Moisson R, Gentry E, Davis S, Dolan T, Rees C (2016) A pilot evaluation of a mindful self-care and resiliency (MSCR) intervention for nurses. Mindfulness 7(3):764-774

5. Dahlgren G, Whitehead M (1991) Policies and strategies to promote social equity in health. Institute for Future Studies,

6. Darawad MW, Nawafleh H, Maharmeh M, Hamdan-Mansour AM, Azzeghaiby SN (2015) The relationship between time pressure and burnout syndrome: a cross-sectional survey among Jordanian nurses. Health 07(01):14-22. https://doi.org/ $10.4236 /$ health.2015.71003

7. Die Träger der Nationalen Präventidesrahmenempfehlungen. https://www. bundesgesundheitsministerium.de/fileadmin/ Dateien/3_Downloads/P/Praeventionsgesetz/ BRE_Fassung_vom_29.08.2018.pdf.Zugegriffen: 2. Febr. 2021

8. Dresing T, Pehl T (2013) Praxisbuch Interview, Transkription \& Analyse. Anleitungen und Reonskonferenz (NPK) (Hrsg) (2018) Bun- gelsysteme für qualitativ Forschende, 5. Aufl. Dr. Dresing und Pehl, Marburg

9. ERBI Software Consult Sozialforschung (2014) MAXQDA 12:. Reference Manual. VERBI Software. Consult. Sozialforschung. GmbH. http:// www.maxqda.com/download/manuals/MAX12_ manual_eng.Zugegriffen:1.Aug. 2020

10. Hand ME, Margolis J, Staffileno BA (2019) Massage chair sessions. Favorable effects on ambulatory cancer center nurses' perceived level of stress, blood pressure, and heart rate. Clin J Oncol Nurs 23:375-381

11. Hersh L, Salzman B, Snyderman D (2015) Health literacy in primary care practice. Am Fam Physician 92(2):118-124

12. Hurley S, Edwards J, Cupp J, Phillips M (2018) Nurses' perceptions of self as role models of health. West J Nurs Res 40(8):1131-1147. https://doi.org/ $10.1177 / 0193945917701396$

13. Institut für Gesundheits- und Sozialforschung (2006) DAK-BGW-Gesundheitsreport. Ambulante Pflege. Arbeitsbedingungen und Gesundheit in ambulanten Pflegediensten. DAK. https://epub. sub.uni-hamburg.de//epub/volltexte/2013/ 24497/pdf/Gesundheitsreport_Ambulante Pflege_2006.pdf.Zugegriffen:1.Aug. 2020

14. KratzerN (2011) Arbeit und Gesundheit im Konflikt: Analysen und Ansätze für ein partizipatives Gesundheitsmanagement. edition sigma,

15. Lary A, Borimnejad L, Mardani-Hamooleh M (2019) The impact of a stress management program on the stress response of nurses in neonatal intensive care units: a quasi-experimental study. J Perinat Neonatal Nurs 33:189-195

16. Lohmann-Haislah A, Wendsche J, Schulz A, Schöllgen I, Pinzon LCE (2020) Erratum zu: Einflussfaktoren und Folgen des Ausfalls gesetzlicher Ruhepausen bei Pflegekräften in Deutschland. Z Arb Wiss 74(2):160. https://doi.org/10.1007/ s41449-020-00195-x

17. Mayring P (2000) Qualitative Content Analysis. Forum Qual Sozialforschung/forum: Qual Soc Res. https://doi.org/10.17169/fqs-1.2.1089

18. McDermott $R$ (2011) Internal and external validity. In: Druckman JN, Green DP, Kuklinski JH, Lupia A (Hrsg) Cambridge handbook of experimental political science. Cambridge University Press, Cambridge, S27-40

19. Mojtahedzadeh N, Neumann FA, Rohwer E, Augustin M, Zyriax B-C, Harth V, Mache S (2020) Betriebliche Gesundheitsförderung in der Pflege. Präv Gesundheitsf. https://doi.org/10. 1007/s11553-020-00800-1

20. Mojtahedzadeh N, Neumann FA, Augustin M, Zyriax B-C, Harth V, Mache S (2020) Das Gesundheitsverhalten von Pflegekräften - aktueller Forschungsstand, Potenziale und mögliche Herausforderungen. Präv Gesundheitsf. https://doi. org/10.1007/s11553-020-00792-y

21. Richter P, Hacker W (1998) Belastung und Beanspruchung: Stress, Ermüdung und Burnout im Arbeitsleben. Asanger,

22. Schulz E (2012) Pflegemarkt: Drohendem Arbeitskräftemangel kann entgegengewirkt werden. DIW Berlin. https://www.diw.de/documents/ publikationen/73/diw_01.c.413133.de/12-51-1. pdf.Zugegriffen: 6. Jan. 2020

23. Statistisches Bundesamt (2019) Bevölkerung im Wandel. Ergebnisse der 14. koordinierten Bevölkerungsvorausberechnung. https://www. destatis.de/DE/Presse/Pressekonferenzen/2019/ Bevoelkerung/statement-bevoelkerung.pdf. Zugegriffen: 1. Aug. 2019 
24. Tausch AP, Menold N (2016) Methodological aspects of focus groups in health research: results of qualitative interviews with focus group moderators. Glob Qual Nurs Res. https://doi.org/ $10.1177 / 2333393616630466$

25. Techniker Krankenkasse (2019) Gesundheitsreport 2019. Pflegefall Pflegebranche? So geht's Deutschlands Pflegekräften

26. Tong A, Sainsbury P, Craig J (2007) Consolidated criteria for reporting qualitative research (COREQ): a 32-item checklist for interviews and focus groups. Int J Qual Health Care 19(6):349-357. https://doi. org/10.1093/intqhc/mzm042

27. Voltmer E, Wingenfeld K, Spahn C, Driessen M, SchulzM (2013) Work-related behaviour and experience patterns of nurses in different professional stages and settings compared to physicians in Germany. Int J Ment Health Nurs 22(2):180-189. https://doi.org/10.1111/j.1447-0349.2012. 00855.x

28. Wendsche J, Hacker W, Wegge J (2017) Understaffing and registered nurses' turnover: the moderating role of regular rest breaks. Ger J Hum Resour Manage 31(3):238-259

29. Wirth T, KozakA, Schedlbauer G, Nienhaus A (2016) Health behaviour, health status and occupational prospects of apprentice nurses and kindergarten teachers in Germany: a cross-sectional study. J Occup Med Toxicol 11:26. https://doi.org/10. 1186/s12995-016-0116-7

30. Zeiher J, Duch M, Kroll LE, Mensink GBM, Finger JD, Keil T (2020) Domain-specific physical activity patterns and cardiorespiratory fitness among the working population: Findings from the cross-sectional German Health Interview and Examination Survey. BMJ Open 10(4):e34610. https://doi.org/10.1136/bmjopen-2019-034610 